\title{
Time-Resolved Diagnostics of Single-Wall Carbon Nanotube Synthesis by Laser Vaporization
}

\author{
Alex A. Puretzkya, David B. Geohegan, C. Henrik Schittenhelm, Xudong Fan, \\ Michael A. Guillorn \\ Oak Ridge National Laboratory, Oak Ridge, TN 37831 \\ ${ }^{a}$ Dept. of Materials Science and Engineering, University of Tennessee
}

\begin{abstract}
Three questions important to nanosecond laser ablation synthesis of single wall carbon nanotubes (SWNT) have been addressed using in situ spectroscopic diagnostics: determining the temperature of the nanoparticles within the propagating plume at different times after ablation, monitoring the aggregation of the nanoparticles in the plume, and measuring the growth rates of the SWNTs. Short SWNTs were synthesized using nanosecond Nd:YAG-laser ablation of a C$\mathrm{Ni}$-Co target inside a high-temperature laser vaporization reactor by controlling and restricting the growth times. The time spent by the plume inside the oven was varied by positioning the target at various locations and imaging the plume using Rayleigh scattered light induced by a 308-nm XeCl laser. Statistical analysis of the short SWNT length distribution was performed using TEM images. The upper and lower limits of the growth rates of SWNTs were estimated as 0.6 and $5.1 \mu \mathrm{m} / \mathrm{s}$. The particle temperature within the propagating plume was measured at different times after ablation through time-resolved measurements of the plume's blackbody emission. The onset of SWNT growth was estimated based on the time when the particle temperature drops below the eutectic temperature for $\mathrm{C} / \mathrm{Co}, \mathrm{C} / \mathrm{Ni}$. For the first time, absorption spectroscopy was employed to study the aggregation of carbon nanoparticles in the propagating plume. It was shown that the aggregation rate increases rapidly at lower oven temperatures. A general picture of SWNT growth by laser ablation based on imaging, spectroscopy, and pyrometry of ejected material at different times after ablation is discussed.
\end{abstract}

Keywords: carbon nanotubes, laser ablation, spectroscopic diagnostics
a) Corresponding author. Tel.: +1-865-574-5497;
fax:+1-865-576-3676. E-mail address: 1ap@ ornl.gov 


\section{Introduction}

Single wall carbon nanotubes exhibit remarkable electronic and structural properties, which promise to revolutionize various application areas, from nanoscale electronics to ultralightweight structural materials [1]. Laser vaporization is one of the best methods to grow highquality, high-purity SWNTs. As typically employed, each laser shot vaporizes a small amount of material $\left(\sim 10^{16}\right.$ carbon atoms and $\sim 10^{14}$ metal catalyst atoms, e.g., $\sim 1$ at $\% \mathrm{Ni}, \mathrm{Co}, \mathrm{Fe}, \mathrm{Y}$, etc.) inside an oven ( 1200 C) into 500 Torr of gently flowing inert gas [2,3]. On a single laser shot [4], the ejected material self-assembles to form a high volume fraction of SWNTs, which can be up to 10 microns in length $[3,5]$.

Since the first introduction of laser vaporization process in 1995 [2] researchers attempted optimization by manipulating numerous experimental parameters, e. g., laser parameters (energy fluence, peak power, repetition rate, $\mathrm{cw}$ vs. pulsed) [4, 6-9], target composition [10,11], carrier gas flow rate and pressure [7, 12], ambient temperature [7, 13], etc. These studies provided a reasonable optimization of the processs and created many speculations about the growth mechanism.

Unfortunately, the growth of SWNTs is not controlled or fully understood. Thus, their potential technological applications, which depend on the atomic-scale structure (chirality), growth rates and the possibility of large-scale production are affected by this lack of the fundamental understanding.

The first attempts to perform in situ spectroscopic studies during SWNT growth were based on the observation of the luminous laser plasma at early times after nanosecond Nd:YAG laser ablation $(0-80 \mu \mathrm{s})$ [14] and after long-pulse $\mathrm{CO}_{2}$-laser vaporization at 25-1200 C [15]. These measurements were limited to times while the ablated material is still quite hot.

Our approach to understanding the growth mechanism and optimizing the yield of SWNTs is to combine laser vaporization and in situ diagnostics [16-18]. Recently, we used laser-induced emission, gated intensified charge-coupled device (ICCD) imaging, and optical spectroscopy to probe the ablated material in a long time interval after ablation (from 0 to a few seconds) [16-18]. In this work the plume of ejected material was followed for several seconds after 8ns-Nd:YAG-laser ablation pulse using the combined imaging and spectroscopy of Co atoms, $\mathrm{C}_{2}$, and $\mathrm{C}_{3}$ molecules, and clusters. These spectroscopic diagnostics showed that 
nanotube growth occurs within a vortex ring plume during the long periods of time $(\sim 1 \mathrm{~s})$ when the atomic molecular vapor already condensed into clusters. The time for conversion of atomic and molecular species to clusters was estimated as $\sim 200 \mu$ s for carbon and $\sim 2 \mathrm{~ms}$ for cobalt at $1100 \mathrm{C}$. We concluded that SWNT grow by condensed phase conversion from feedstock of carbon and metal nanoparticles. This conclusion was confirmed by our recent work on ex situ high temperature (900-1200 C) annealing of the precursor material generated by time-restricted laser ablation and containing short SWNTs $(<200 \mathrm{~nm})$, amorphous carbon, and metal nanoparticles [19].

The main results of these studies were later confirmed by Kokai et al [20] who used similar time-resolved imaging and spectroscopy approach based on in situ measurements of laser plume emission and light scattering from the propagating plume.

In situ monitoring of catalyst $\mathrm{Ni}$ atoms by laser induced fluorescence near the target region (1-3 mm) was performed by De Boer et al [21], who concluded that Ni stays in atomic vapor phase for several milliseconds after ablation. This is consistent with our measurements on Co [16-18].

Prior to these diagnostic studies very little was known about where, when, and at what rate the growth occurred. Recently, despite diagnostic investigations, estimates for growth rates of SWNTs by laser vaporization inside the hot oven environment range over five orders of magnitude, from $\sim 1 \mu \mathrm{m} / \mathrm{s}$ up to $\sim 15 \mathrm{~cm} / \mathrm{s}[22,23]$.

In this paper detailed information on the growth environment of SWNTs during the early stages of their development is provided with new estimates of size and temperature of the aggregated nanomaterial which propagates inside the oven reactor a few milliseconds after laser vaporization. Optical absorption and emission spectroscopy techniques are described for these measurements. These measurements are correlated with oven temperature profiles and Rayleighscattering imaging of the propagating nanomaterial to restrict growth times available at known temperatures. Using length distributions of SWNTs produced under these well-defined conditions, the upper and lower limits of the growth rates of SWNTs were estimated as 0.6 and $5.1 \mu \mathrm{m} / \mathrm{s}$ for the typical nanosecond laser vaporization conditions used in this study.

In conclusion, a comprehensive picture of SWNT growth is presented which combines our spatial and temporal measurements of plume composition, temperature, and particle size. 
These new results further support our previous studies which indicate that SWNT grow over extended times ( $>100 \mathrm{~ms}$ to seconds) by a condensed phase conversion process.

\section{Experimental}

The SWNT growth setup is similar to that described in Refs. [17, 18]. It consists of a quartz tube ( 2 in. diameter, 24 in. length) mounted inside a hinged tube furnace (12 in. length) that can operate at maximum temperature of $1200 \mathrm{C}$. The quartz tube was O-ring sealed to standard 4.5-in. conflat vacuum components. The ablation and probe laser beams entered the quartz tube through the same Suprasil window, which was mounted in a vacuum flange. A dichroic mirror was used to pass the ablation and probe laser beams coaxially. The furnace was equipped with a rectangular quartz window (10 in. length, 1 in. width, Suprasil 1) for spectroscopic diagnostics of ablated material inside the furnace. A temperature profile along the axis of the quartz tube was measured by introducing a thermocouple coaxially with the quartz tube. In this case the Suprasil laser-entry window was replaced with a mount for the thermocouple. The end of the thermocouple could be positioned at any point at the tube axis.

Argon gas was introduced around the quartz window and was controlled at $100 \mathrm{sccm}$ to maintain a 500 Torr pressure. The gas was pumped out through a needle valve downstream of a brass water-cooled collector, which was inserted into the quartz tube and positioned just outside the furnace.

A 1-in. diameter graphite target containing 1 at \% each of $\mathrm{Ni}$ (Alfa, 2.2-3.0 $\mu \mathrm{m}, 99.9 \%$ ) and Co (Alfa, 1-6 um, 99.8\%) powders was prepared with carbon cement (Dylon GC). The target was screwed onto a 0.25 in. diameter graphite rod and was rotated during operation. This rod was mounted along the tube axis through a hole in the collector.

The ablation laser (1.06 $\mu \mathrm{m} \mathrm{Nd:YAG,} 300 \mathrm{~mJ}, 8 \mathrm{~ns}$ FWHM pulse) beam was focused to a $4 \mathrm{~mm}$-diameter, donut-shaped spot on the target. The energy density at the target was about 3 $\mathrm{J} / \mathrm{cm}^{2}$. A gated ICCD-camera system (Princeton Instruments, $5 \mathrm{~ns}$ minimum gate, 200-820 nm spectral range) was used to perform scattering imaging of the ablation plume using a defocused XeCl-laser pulse $\left(308 \mathrm{~nm}, 30 \mathrm{~ns}\right.$ FWHM, $\left.6 \mathrm{~mJ} / \mathrm{cm}^{2}\right)$ at different time delays. The $5 \mathrm{~ns}-\mathrm{ICCD}$ gate was set to occur at the peak of the $\mathrm{XeCl}$ laser pulse. 
A 1 in. diameter quartz window located near the center of the furnace at the opposite side of the rectangular window allowed absorption spectroscopy of the ejected material. In this case a collimated beam from a pulsed Xe-lamp ( 1 $\mu$ s FWHM) was passed through the plume and its extinction spectrum was measured at different times after ablation. The absorption spectra were recorded with a $0.3 \mathrm{~m}$ spectrometer (Acton VM-503) equipped with an intensified, gated diode array (Princeton Instruments IRY-700 RB).

\section{Growth of short SWNTs}

Growth rates of SWNTs were estimated by measuring the length distribution of SWNTs for known growth times. However, reliable estimates of SWNT lengths can only be performed for short nanotubes where the ends of the nanotubes can be clearly determined in TEM pictures. To limit the time available for nanotube growth, the target was located close to the front edge of the furnace $(\mathrm{d}=10 \mathrm{~cm})$ such that, after a known time spent at relatively uniform temperature, the plume would be thermophoretically drawn as rapidly as possible out the front of the oven to deposit upstream on the wall of the quartz tube. Of utmost importance, however, was to assure that the plume spent a guaranteed time $(10-20 \mathrm{~ms})$ in the uniform-temperature region of the oven before the rapid cooling at the oven edge. Rayleigh-scattering images of the plume (in association with the measured temperature profiles of the oven) were used to provide this known growth time at uniform temperature.

Figure 1 shows the oven temperature profiles measured at three different temperatures, 780,960 , and $1100 \mathrm{C}$ at the center of the furnace. The temperature distribution along the quartz tube axis is uniform near the center of the tube and drops rapidly at both ends. To define the uniform temperature zone we fitted the gradient region of the temperature profiles with a straight line and used a cross point to this line and the plateau to estimate the lower limit of the uniform temperature zone. The upper temperature was defined by the target position (see inset in Fig. 1). Using this procedure we defined a uniform temperature zone located approximately between 5 and $10 \mathrm{~cm}$ from the front oven edge. The temperature within this zone changes from 760 to 715 ${ }^{\circ} \mathrm{C}(\Delta \mathrm{T} / \mathrm{T}=5.9 \%) ; 950-900{ }^{\circ} \mathrm{C}(\Delta \mathrm{T} / \mathrm{T}=5.6 \%)$; and $1100-1050{ }^{\circ} \mathrm{C}(\Delta \mathrm{T} / \mathrm{T}=4.6 \%)$ for three

different temperatures used to grow short SWNTs. The gradient temperature zone located 
approximately between 0 and $5 \mathrm{~cm}$ (see Fig. 1) can be characterized by rapid temperature drop $70{ }^{\circ} \mathrm{C} / \mathrm{cm}$. This temperature zone was used to stop the growth of SWNTs.

To find the positions of the plume inside and outside the furnace we used Rayleigh scattering imaging. Figure 2 shows images of the plume at 2 and $15 \mathrm{~ms}$ after ablation inside the furnace and at 200, 250, and $500 \mathrm{~ms}$ when the plume exits the furnace. The detailed description of the plume dynamics during SWNT growth was given in Refs. [17, 18]. Initially the plume generates a strong shock front and traps itself between this shock front and the target. The forward propagating plume sets the surrounding background gas in motion in such a way that it flows around the plume and forms vortices. These vortices trap the ejected material at later times after ablation.

In the uniform temperature zone the shape of the vortex ring does not change much when it propagates forward. The diameter of the vortex ring usually increases slowly with time [17, 18]. When the vortex ring approaches the temperature gradient zone its plane tilts relative to the tube axis and the ring elongates along this axis [17, 18]. At $200 \mathrm{~ms}$ the plume exits the furnace in this tilted orientation to deposit onto the upper surface of the quartz tube. The ejected material spends approximately 10-20 ms at the uniform temperature and 100-200 ms in the steep gradient zone.

Using this approach we can grow short SWNTs. Figure 3a shows TEM images of short SWNTs synthesized at furnace temperature $\sim 760 \mathrm{C}$. This image demonstrates that many short tubes sprout from catalyst nanoparticles represented by small black dots in Fig. 3a. In most cases we can easily see the ends of these short tubes and measure their length. The length distribution of short SWNTs was fitted with a logarithmic normal distribution (Fig. 3b). At higher furnace temperatures the maximum of the nanotube length distribution shifts to longer lengths and the width of the distribution increases. At $760 \mathrm{C}$ the most probable value of length is $35 \mathrm{~nm}$ and $90 \%$ of all tubes are shorter than $90 \mathrm{~nm}$. These values are $74 \mathrm{~nm}$ and $170 \mathrm{~nm}$ for $960 \mathrm{C} ; 77 \mathrm{~nm}$ and $240 \mathrm{~nm}$ for $1100 \mathrm{C}$.

\section{Measurements of particles temperature inside the propagating vortex ring plume}

It is important to know the temperature of the particles in the propagating plume and to correlate this temperature with the time after ablation. First, using this correlation we can 
estimate when SWNTs start to grow, since by this time the temperature should not exceed the eutectic temperature for metal catalyst nanoparticles. It was shown experimentally that the yield of SWNT drops rapidly at $\mathrm{T}>\mathrm{T}_{\text {eut }}(\mathrm{C} / \mathrm{Co}, \mathrm{C} / \mathrm{Ni})$ [24]. Second, to limit the growth times and to estimate the growth rates of SWNTs it is necessary to determine how fast the species within the plume reach the ambient furnace temperature.

To estimate the temperature of carbon particles inside the propagating plume we measured their blackbody emission (incandescence) spectra at different times and positions in the furnace (Fig. 4a). The plume emission spectra were fitted with the Planck blackbody function. The intensity of the blackbody emission from a particle of radius $a$ at a wavelength $\lambda$ into interval $\Delta \lambda$ is given by

$$
\mathrm{I}(\lambda, a)=\varepsilon(\lambda, a) 8 \pi^{2} a^{2} \mathrm{c}^{2} \mathrm{~h} \Delta \lambda / \lambda^{5}\left[\exp \left(\mathrm{hc} / \lambda \mathrm{k}_{\mathrm{B}} \mathrm{T}\right)-1\right]
$$

where $\varepsilon(\lambda, a)$ is the spectral emissivity and $\mathrm{T}$ is the absolute temperature of the particle. For small particles $(a<0.3 \lambda / 2 \pi) \varepsilon(\lambda, a) \propto 1 / \lambda$ and $\mathrm{I}(\lambda, a) \propto 1 / \lambda^{6}$. For larger particles we can assume that $\varepsilon=$ const that gives, $\mathrm{I}(\lambda, a) \propto 1 / \lambda^{5}$. It should be mentioned that an ICCD detector counts photons, therefore the number of photons per unit time will be proportional to $1 / \lambda^{4}$ in the case of the constant emissivity $\varepsilon$.

Fig. $4 \mathrm{~b}$ shows an example of the blackbody fit of the emission spectrum of the carbon particles in the plume measured at $\mathrm{t}=1 \mathrm{~ms}$ after ablation assuming that $\varepsilon=$ const. This fit gives the plume temperature of $1488 \mathrm{C}$. Figure 5a shows the temperature of the particles within the plume versus the time after ablation. The temperature of the plume approaches the ambient temperature $\left(\mathrm{T}_{\mathrm{abm}}=750 \mathrm{C}\right)$ after approximately $4 \mathrm{~ms}$ after ablation. For $\mathrm{T}_{\mathrm{amb}}=1100 \mathrm{C}$, a plateau is observed at $\mathrm{T} \sim 2500 \mathrm{C}$ in the time interval 0.3-0.7 ms (Fig. 4b). A similar plateau in the plume emission intensity was observed by Suzuki et al [25]. An exothermic process, probably formation of fullerene-like structures, keeps the plume temperature constant from 0.3 to $0.7 \mathrm{~ms}$. In the case of higher ambient temperature $(1100 \mathrm{C})$, it is also takes approximately $4 \mathrm{~ms}$ after ablation for the particles within the plume to cool down to the ambient temperature.

\section{Measurements of growth rates of SWNT}


Using the measured values of the most probable length of SWNTs (Fig. 3b) and the times the ablated material spent in the uniform temperature zones, and taking into account the short time ( $\sim \mathrm{ms}$ ) required to cool the particles within the plume down to the ambient temperature we can estimate the growth rates of SWNTs. The estimated growth rates are listed in Table 1 for the three ambient temperatures used in this study.

We estimated the lower and the upper limits of the growth rates using two different criteria. The lower limit was estimated on the basis of the minimum temperature at which the SWNT can grow. Bandow et al [13] estimated that the yield of SWNT produced by nanosecond laser vaporization was about $3-5 \%$ at $\mathrm{T}=780 \mathrm{C}$. They did not find any tubes at room temperature. Sen et al [26] concluded that the lowest threshold temperature for nanotube growth in nanosecond laser ablation process is about $850 \mathrm{C}$. Our study shows that short SWNTs can be synthesized at $750 \mathrm{C}$. To estimate the lower limit of the growth rate we assumed that the minimum temperature for SWNT growth in nanosecond laser vaporization process is about 700 $\mathrm{C}$, and estimated the times the ablated material spent at $\mathrm{T}>700 \mathrm{C}$ (Table 1). The upper limit of the growth rate was estimated based on the time the vaporized material spent in the uniform temperature zone. Using these assumptions and assuming that the growth rate is constant we can estimate the growth rate of SWNTs as $1-5 \mu \mathrm{m} / \mathrm{s}$.

\section{In situ absorption spectroscopy of carbon nanoparticles: estimating particle size}

To estimate the size of carbon nanoparticles in the plume we used absorption spectroscopy. In this case a beam from a pulsed Xe-lamp was passed through the plume and its extinction spectrum (scattering plus absorption) was measured (Fig. 6a). The particle size was estimated from the shape of the extinction spectrum. For small particles $(2 \pi \mathrm{a}<<\lambda$, Rayleigh criterion: $2 \pi \mathrm{a}<0.3 \lambda$ ) the absorption and scattering cross-sections are given by [27]

$$
\mathrm{Q}_{\mathrm{abs}}=4(2 \pi a / \lambda) \operatorname{Im}\left[\left(\mathrm{m}^{2}-1\right) /\left(\mathrm{m}^{2}+2\right)\right], \quad \mathrm{Q}_{\mathrm{sca}}=(8 / 3)(2 \pi a / \lambda)^{4}\left|\left(\mathrm{~m}^{2}-1\right) /\left(\mathrm{m}^{2}+2\right)\right|,
$$

where $a$ is the particle radius, $m$ is the complex index of refraction, $m=n-k i$. If $m$ is only a weak function of $\lambda$ over the measured wavelength range, than $\mathrm{Q}_{\mathrm{abs}} \propto 1 / \lambda, \mathrm{Q}_{\mathrm{sca}} \propto 1 / \lambda^{4}$, and there is no size information in the shape of the extinction spectra. 
For larger particles $(a>0.05 \lambda)$ the shape of the absorption/extinction spectrum is sensitive to the particle size. In this case the particle size can be estimated from the shape of the extinction spectra using Mie theory for spherical particles and the computer code given in Ref. [27], and the complex index of refraction, $m(\lambda)$, for carbon soot particles given in Ref. [28].

Figures $6 \mathrm{~b}, \mathrm{c}$ show the extinction spectra of the laser generated plumes measured at two different ambient temperatures, $760 \mathrm{C}$ and $1000 \mathrm{C}$, approximately $1 \mathrm{~ms}$ after ablation. The set of extinction spectra, calculated based on Mie theory for spherical particles, having different radii is shown for comparison. One can see that at $760 \mathrm{C}$ oven temperature the shape of the measured spectrum is approximately fitted by the curve calculated for $a=80 \mathrm{~nm}$. This means that by $1 \mathrm{~ms}$ the clusters aggregated to form relatively large aggregates. The spectrum measured at $1100 \mathrm{C}$ is fitted fairly well by the extinction spectum calculated for $a=20 \mathrm{~nm}$. This particle size is close to obeying the Rayleigh criterion $(\mathrm{a} \sim 14 \mathrm{~nm}$ for $\lambda=300 \mathrm{~nm})$ and one can conclude that in this case the size of the particles is smaller than $20 \mathrm{~nm}$. The measured spectra have the same shape up to $4 \mathrm{~ms}$ after ablation. Summarizing the absorption measurements we can conclude that carbon nanoparticles aggregate much slower at higher ambient temperatures.

It should be mentioned that to deduce the particles size we used Mie theory for the spherical particles and the proper particle refractive index. In reality clusters form polydisperse fractal aggregates. The interpretation of absolute angular light scattering extinction measurements for such aggregates is given in Ref. [29], were it was shown that volume-equivalent single particle models (Rayleigh/Mie) overestimated the particle radius by a factor of $\sim 3$.

Using the absorption spectroscopy we can also monitor the atomic catalyst. Figure 7 shows the absorption spectrum measured at $600 \mu \mathrm{s}$ after ablation at $1100 \mathrm{C}$. The peaks near $350 \mathrm{~nm}$ can be assigned to atomic Co and Ni lines. Unfortunately, the low signal to noise level in this measurement did not allow us to make the exact assignment of these lines. These atomic lines are superimposed on the broad absorption spectrum from carbon clusters. The presence of the strong absorption continuum from carbon clusters at $600 \mu \mathrm{s}$ after ablation is consistent with our previous studies based on laser induced fluorescence (LIF), which show that the atomic/molecular carbon converts to clusters very rapidly during the first $200 \mu \mathrm{s}$, but the catalyst metal stays in the atomic phase much longer and disappears approximately after 2 ms after ablation [17,18]. In addition to LIF spectroscopy and imaging to observe the metal catalyst 
atomic species, the absorption spectroscopy can be a useful tool to estimate an absolute concentration of metal catalyst atoms in the propagating $\mathrm{C} / \mathrm{Co} / \mathrm{Ni}$ plume.

\section{Conclusions: the sequence of events in laser vaporization leading to SWNT growth.}

We can deduce a comprehensive picture of the events in laser ablation leading to SWNT growth by combining the results of the temperature and particle size measurements of the particles within the propagating plume with the results of our previous studies on plume dynamics and spectroscopy, described in references [17, 18].

Figure 8 summarizes the results of our in situ spectroscopic diagnostics using time, spatial, and temperature coordinates. Initially a Nd:YAG laser pulse produces atomic/molecular vapor containing $\sim 5 \cdot 10^{16}$ carbon and $\sim 10^{14} \mathrm{Ni} / \mathrm{Co}$ atoms as estimated by weighing the $\mathrm{C} / \mathrm{Ni} / \mathrm{Co}$ target before and after laser ablation. The evaporated material stays in the vapor phase until approximately $100 \mu$ s after ablation [17,18]. During this time the laser plasma is very hot and emission from excited atoms and molecules dominate laser-induced emission from ground state species. The laser plasma cools rapidly, increasing the populations of the atomic and molecular ground states as indicated by LIF measurements.

To observe the plume of ejected material at $\mathrm{t}>200 \mu \mathrm{s}$ we used laser induced incandescence and Rayleigh scattering from clusters and nanoparticles. By that time the plume becomes substantially nonuniform as it can be seen from the Rayleigh scattering images (Figs. 2, 8) demonstrating pronounced turbulent structure within the plumes. At later times (> $2 \mathrm{~ms})$ the plume aquires a characteristic vortex ring shape that still has a highly turbulent substructure (Figs. 2, 8).

Images of the plume and LIF spectra show that carbon condenses and forms clusters by $\mathrm{t}$ $=200 \mu$ s after ablation, while the metal catalyst atoms condense much later. The ground-state atomic Co population is maximum at $\mathrm{t} \sim 0.8 \mathrm{~ms}$, and then $\sim 90 \%$ of cobalt atoms condence into clusters by $\mathrm{t} \sim 2 \mathrm{~ms}[17,18]$. The absorption spectra measured at $\sim 0.6 \mathrm{~ms}$ after ablation (Fig. 7) show that the atomic $\mathrm{Ni}$, Co lines are superimposed on the strong background absorption from carbon nanoparticles. Thus, by $\sim 2 \mathrm{~ms}$ the great majority of both carbon and metal vapor have condensed into clusters and nanoparticles, a large fraction of which are somehow subsequently converted into SWNTs. The mobility of these nanoparticles clearly depends upon their size, 
hence the size and aggregation rate of these nanoparticles were measured during the crucial period between $\mathrm{t}=1-4 \mathrm{~ms}$ (see below).

The Mie theory interpretation of the extinction spectra (absorption plus scattering, Fig. 6c) shows that the size of the carbon particles within the plume at these times does not exceed 20 $\mathrm{nm}$ at ambient temperature of $\sim 1100 \mathrm{C}$. However, at lower ambient temperatures ( 760 C) a much larger aggregates were observed. Using Mie theory for spherical particles the size of these aggregates was estimated as $\sim 80 \mathrm{~nm}$ This rapid aggregation of carbon nanoparticles at lower processing temperatures results in a decrease in mobility and could be one of the reasons for the rapid decrease of SWNT yields at lower ambient temperatures.

The temperature measurements in this study permit an estimate for the onset of SWNT growth. At $\mathrm{t}=2 \mathrm{~ms}$ the plume temperature is $\sim 1400 \mathrm{C}$, just above the $\mathrm{Ni} / \mathrm{C}$ and $\mathrm{Co} / \mathrm{C}$ eutectic temperatures. Since it is well known that the yield of SWNTs drops rapidly at $\mathrm{T}>\mathrm{T}_{\text {eut. }}[7,24]$, we can estimate the onset of SWNT growth as $\sim 2 \mathrm{~ms}$ for our experimental conditions. By this time, we have shown that the majority of the ejected vapor has already condenced into clusters and nanoparticulate aggregates, so SWNT nucleation appears to occur in this environment.

In order to confirm where the majority of SWNT growth occurs and estimate growth rates, plume imaging and steep temperature gradients near the end of the furnace were employed to restrict the growth times of SWNTs to about 15-20 ms. Very short SWNTs forming thin bundles consisting of a few tubes were found to have most probable lengths of 35-77 nm depending on the processing ambient temperature, 760-1100 C. This permits upper and lower limits for the growth rate of SWNTs to be estimated as $\sim 0.6$ and $5.1 \mu \mathrm{m} / \mathrm{s}$, respectively. However, the main conclusions of these time-resolved growth studies are that the majority of SWNT growth occurs for times longer than $20 \mathrm{~ms}$ after laser vaporization, by the conversion of condense phase carbon and metal catalyst clusters and nanoparticles.

\section{Acknowledgments}

The authors gratefully acknowledge the assistance of P.F. Britt and S.J. Pennycook. This research was sponsored by the U.S. Department of Energy under contract DE-AC05-00OR22725 with the Oak Ridge National Laboratory, managed by UT-Battelle, LLC and the LaboratoryDirected Research and Development Program at ORNL. 


\section{References}

1. B.T Yakobson and R.E. Smalley, American Scientist, 85 (1997) 324 .

2. T Guo, P. Nikolaev, A. Thess, D.T. Colbert, R.E. Smalley, Chem. Phys. Lett. 236 (1995) 419.

3. A. Thess, R. Lee, P. Nikolaev, H. Dai, P. Petit, J. Robert, C. Xu, Y.H. Lee, S.G. Kim, A.G. Rinzler, D.T. Colbert, G.E. Scuseria, D. Tomanek, J.E. Fisher, R.E. Smalley, Science 273 (1996) 483 .

4. M. Yudasaka, T. Ichihashi, T. Komatsu, S. Iijima, Chem. Phys. Lett. 299 (1999) 91.

5. A.G. Rinzler, J. Liu, H. Dai, P. Nikolaev, C.B. Huffman, F.J. Rodriguez-Macias, P.J. Boul, A.H. Lu, D. Heymann, D.T. Colbert, R.S. Lee, J.E. Fisher, A.M. Rao, P.C. Eklund, R.E. Smalley, Appl. Phys. A 67 (1998) 29.

6. M. Yudasaka, T. Ichihashi, S. Iijima, J. Phys. Chem. B 102 (1998) 10201.

7. A.A. Gorbunov, R. Friedlein, O. Jost, M.S. Golden, J. Fink, W. Pompe, Appl. Phys. A 69 (1999) S593.

8. A.C. Dillon, P.A. Parilla, J.L. Alleman, J.D. Perkins, M.J. Heben, Chem. Phys. Lett. 316 (2000) 13.

9. A.C. Dillon, P.A. Parilla, K.M. Jones, G. Riker, M.J. Heben, Mat. Res. Soc. Symp. Proc. Vol. 256 (1998) 403.

10. M. Yudasaka, R. Yamada, N. Sensui, T. Wilkins, T. Ichihashi, S. Iijima, J. Phys. Chem. B 103 (1999) 6224.

11. O. Jost, A.A. Gorbunov, J. Moller, W. Pompe, A. Graft, R. Friedlein, X. Liu, M.S. Golden, J. Fink, Chem. Phys. Lett. 339 (2001) 297.

12. M. Yudasaka, T. Komatsu, T. Ichihashi, Y. Achiba, S. Iijima, J. Phys. Chem. B 102 (1998) 4892.

13. A.S. Bandow, S. Asaka, Y. Saito, A. M. Rao, L. Grigorian, E. Richter, P. C. Eklund, Phys. Rev. Lett. 80 (1998) 3779.

14. S. Arepalli, C.D. Scott, Chem. Phys. Lett. 302 (2001) 139.

15. F. Kokai, K. Takahashi, M. Yudasaka, R. Yamada, T. Ichihashi, and S. Iijima, J. Phys. Chem. B 103 (1999) 4346.

16. D.B. Geohegan, Talk presented at the Fifth International Conference on Laser Ablation, Goettingen, Germany, July 18-23, 1999.

17. A. Puretzky, D.B. Geohegan, X. Fan, S.J. Pennycook, Appl. Phys. Lett. 76 (2000) 182.

18. A.A. Puretzky, D.B. Geohegan, X. Fan, S.J. Pennycook, Appl. Phys. A 70 (2000) 153.

19. D.B. Geohegan, H. Schittenhelm, X. Fan, S.J. Pennycook, A.A. Puretzky, M.A. Guillorn, D.A. Blom, D.C. Joy, Appl. Phys. Lett. 78 (2001) 3307.

20. F. Kokai, K. Takahashi, M. Yudasaka, and S. Iijima, J. Phys. Chem. B 104 (2000) 6777. 
21. G. De Boer, S. Arepalli, W. Holmes, P. Nikolaev, C. Range, C. Scott, J. Appl. Phys. 89 (2001) 5760.

22. S. Arepalli, P. Nikolaev, W. Holmes, B.S. Files, Appl. Phys. Lett. 78 (2001) 1610.

23. C.D. Scott, S. Arepalli, P. Nikolaev, R.E. Smalley, Appl. Phys. A 72 (2001) 573.

24. H. Kataura, Y. Kumazawa, Y. Maniwa, Y. Ohtsuka, R. Sen, S. Suzuki, Y. Achiba, Carbon 38 (2000) 1691.

25. S. Suzuki, R. Sen, H. Yamaguchi, T. Ishigaki, Y. Ohtsuka, Y. Achiba, H. Kataura, "Time and space evolution of emitting carbon nanoparticles: correlation with the formation of fullerenes and carbon nanotubes" (to be published).

26. R. Sen, Y Ohtsuka, T. Ishigaki, D. Kasuya, S. Suzuki, H. Kataura, y. Achiba, Chem. Phys. Lett. 332 (2000) 467.

27.C.F. Bohren, D.R. Huffman, "Absorption and Scattering of Light by Small Particles”, Wiley-Interscience, 1983, p.136.

28. E.A. Rohlfing, J. Chem. Phys. 89 (1988) 6103.

29. U.O. Koylu, Combustion and Flame 109 (1996) 488. 


\section{Figure captions}

Fig. 1. Temperature profiles along the furnace axis measured at three different furnace temperatures: 780,960, and $1100 \mathrm{C}$ at the center of the furnace. The inset at the bottom shows target and window edge positions relative to the oven edge at $d=0$. The open circle separates steep gradient and uniform temperature zones.

Fig. 2. Images of Rayleigh-scattered light (308 $\mathrm{nm}$ XeCl-laser, $26 \mathrm{~ns} F W H M, 6 \mathrm{~mJ} / \mathrm{cm}^{2}$ ) from $\mathrm{C} / \mathrm{Ni} / \mathrm{Co}$ plume during synthesis of short SWNTs at 2 and $15 \mathrm{~ms}$ after ablation inside the furnace and at 200, 250, and $500 \mathrm{~ms}$ when the plume exits the furnace. The temperature at the center of the furnace was $780 \mathrm{C}$. A Nd:YAG-laser vaporizes a $\mathrm{C} / \mathrm{Ni} / \mathrm{Co}$ target inside a 2-in. diameter quartz tube in 500 Torr Ar (flowing to the right at $100 \mathrm{sccm}$. Each image represents a different ablation event ( $5 \mathrm{~ns}$ gate width, opened simultaneously with the probing XeCl-laser pulse).

Fig. 3. (a) TEM images of short SWNTs grown by laser vaporization at $760 \mathrm{C}$. Note that the temperature was measured inside the quartz tube at the target position. (b) Length distribution of short SWNTs generated by the plume spending < $200 \mathrm{~ms}$ inside the furnace at $760 \mathrm{C}$. The curve represent a logarithmic normal distribution fit.

Fig. 4. (a) Schematic of the temperature measurements of the particles inside the propagating plume. (b) Experimental blackbody emission spectrum of carbon nanoparticles within the plume measured at $1 \mathrm{~ms}$ after ablation (dark curve) and fit to Planck's Law yielding the temperature of $1488 \mathrm{C}$ (light curve).

Fig. 5. Temperature of carbon particles inside the propagating plume versus time after ablation measured at different ambient temperatures: (a) $760 \mathrm{C}, \varepsilon(\lambda$, a $)=$ const., and (b) $1100 \mathrm{C}, \varepsilon(\lambda$, a) $=$ const. (solid circles), and $\varepsilon(\lambda, a) \propto 1 / \lambda$ (open circles).

Fig. 6. (a) Schematic of the absorption measurements in the propagating plume. Experimental extinction spectrum (bold curve) and the set of extinction spectra calculated on the basis of Mie theory for spherical particles at $\mathrm{t} \sim 1 \mathrm{~ms}$ at (b) $760 \mathrm{C}$ and (c) at $1000 \mathrm{C}$ oven temperatures.

Fig. 7. Absorption spectrum of $\mathrm{C} / \mathrm{Co} / \mathrm{Ni}$ plume measured at $0.6 \mathrm{~ms}$ after ablation. The inset shows the atomic $\mathrm{Co}$, Ni spectrum obtained by subtraction of the continuous background spectrum from carbon nanoparticles. The set of the most intense atomic $\mathrm{Co}$, Ni absorption lines is shown for the tentative assignment of the experimental peaks. 
Fig. 8. A compendium of the results from the in situ imaging and spectroscopic diagnostic investigations of SWNT growth inside a hot oven. Actual images of the laser plasma $(t<200$ $\mu \mathrm{s})$ and Rayleigh-scattering images of the plume ( $\mathrm{t}>200 \mu \mathrm{s}$ ) are shown vs. time (scale in $\mathrm{cm}$ at right). During the first $100 \mu \mathrm{s}$ after ablation, the laser plasma is very hot, and emission from excited atoms and molecules dominate LIF from ground state species. Ground state populations then peak, and subsequently disappear due to condensation. Images and LIF spectra show that carbon condenses by $\mathrm{t}=200 \mu \mathrm{s}$ after ablation, while the metal catalyst atoms condense much later. The ground-state atomic Co population is maximum at $\mathrm{t}=1 \mathrm{~ms}$, and then condenses by $\mathrm{t}=$ $2 \mathrm{~ms}$. By $\mathrm{t}=2 \mathrm{~ms}$, no LIF is detectable, only LIL and RS from clusters and nanoparticles (i.e. nearly all atoms and molecules have converted into clusters and nanoparticles, as evidenced by the vortex ring structure of the plume). At $\mathrm{t}=2 \mathrm{~ms}$, the plume temperature is $\sim 1400 \mathrm{C}$, just above the $\mathrm{Ni} / \mathrm{C}$ and $\mathrm{Co} / \mathrm{C}$ eutectic temperatures (note that $\mathrm{Ni} / \mathrm{C}$ and $\mathrm{Co} / \mathrm{C}$ eutectic temperatures are slightly lower for nanoparticles compared to the bulk material). By $\mathrm{t}=4 \mathrm{~ms}$, the plume has thermalized to the oven temperature. If growth is stopped at $\mathrm{t} \sim 25 \mathrm{~ms}$, only short nanotubes are found ( $<240 \mathrm{~nm}$ in length), indicating that the majority of growth takes place over much longer times, during the $\sim 10$-second traversal time through the oven, past the target, to the collector. These results indicate that the majority of growth occurs from condensed clusters and nanoparticles of carbon and metal catalyst in contact with one another. 
Table.1. The lower and the upper limits of the growth rates of SWNTs synthesized by nanosecond laser vaporization of a $\mathrm{C} / \mathrm{Co} / \mathrm{Ni}$ target.

\begin{tabular}{|l|l|l|l|l|l|l|l|}
\hline \multicolumn{4}{|c|}{ Lower limit of growth rate } & \multicolumn{3}{c|}{ Upper limit of growth rate } \\
\hline $\begin{array}{l}\text { Oven temp. } \\
\text { range, }{ }^{\circ} \mathrm{C}\end{array}$ & $\begin{array}{l}\text { Time at } \\
\mathrm{T}>700^{\circ} \mathrm{C}\end{array}$ & $\begin{array}{l}\text { Most } \\
\text { probable } \\
\text { length }\end{array}$ & $\begin{array}{l}\text { Growth } \\
\text { rate }\end{array}$ & $\begin{array}{l}\text { Uniform } \\
\text { oven temp. } \\
\text { range, }{ }^{\circ} \mathrm{C}\end{array}$ & $\begin{array}{l}\text { Time in } \\
\text { uniform zone } \\
\mathrm{T}>700^{\circ} \mathrm{C}\end{array}$ & $\begin{array}{l}\text { Most } \\
\text { probable } \\
\text { length }\end{array}$ & $\begin{array}{l}\text { Growth } \\
\text { rate }\end{array}$ \\
\hline $750-700$ & $25 \mathrm{~ms}$ & $35 \mathrm{~nm}$ & $1.4 \mu \mathrm{m} / \mathrm{s}$ & $750-715$ & $20 \mathrm{~ms}$ & $35 \mathrm{~nm}$ & $1.8 \mu \mathrm{m} / \mathrm{s}$ \\
\hline $900-700$ & $100 \mathrm{~ms}$ & $74 \mathrm{~nm}$ & $0.7 \mu \mathrm{m} / \mathrm{s}$ & $950-900$ & $15 \mathrm{~ms}$ & $74 \mathrm{~nm}$ & $5.0 \mu \mathrm{m} / \mathrm{s}$ \\
\hline $1100-700$ & $120 \mathrm{~ms}$ & $77 \mathrm{~nm}$ & $0.6 \mu \mathrm{m} / \mathrm{s}$ & $1100-1050$ & $15 \mathrm{~ms}$ & $77 \mathrm{~nm}$ & $5.1 \mu \mathrm{m} / \mathrm{s}$ \\
\hline
\end{tabular}

